\title{
Stable and Flexible Side-Entry Stage for Nion STEMs
}

Michael T. Hotz ${ }^{1}$, George Corbin ${ }^{1}$, Niklas Delby ${ }^{1}$, Tracy C. Lovejoy ${ }^{1}$, Gwyn Skone ${ }^{1}$, Jean-Denis Blazit ${ }^{2}$, Mathieu Kociak ${ }^{2}$, Odile Stephan ${ }^{2}$, Marcel Tencé ${ }^{2}$, Henny W. Zandbergen ${ }^{3}$ and Ondrej L. Krivanek ${ }^{1,4}$

1. Nion R\&D, 11511 NE 118th St., Kirkland, WA 98034, USA

2. Laboratoire de Physique des Solides, CNRS, Université Paris Sud XI, F 91405 Orsay, France

3. Department of NanoScience, Delft University of Technology, 2628 CJ Delft, The Netherlands

4. Department of Physics, Arizona State University, Tempe, AZ 85287, USA

Aberration-corrected scanning transmission electron microscopes (STEMs) can resolve single atoms, probe them spectroscopically, and produce elemental and chemical maps with atomic resolution in 2D and 3D. When equipped with a monochromator and operating in an aloof mode, STEMs can record vibrational electron energy loss spectra (vib-EELS) to create nm-scale vibrational maps while avoiding radiation damage. These techniques require excellent short-term (vibration) and long-term (drift) stability of the microscope's sample stage.

Typical side-entry stages are mounted on the side of the microscope in such a way that the sample is connected to the objective lens (OL) polepiece through a $\sim 30 \mathrm{~cm}$ long mechanical path. This means that if the temperature of the microscope (or of the sample rod) changes by just $0.1^{\circ} \mathrm{C}$ then the sample can drift by hundreds of $\mathrm{nm}$. This results in image drift rates of the order of $\mathrm{nm}$ per minute being common with side-entry stages in all but the most stable environments.

In order to minimize these kinds of problems, Nion's cartridge-based sample stage was designed to have an OL polepiece-to-sample mechanical path of $10 \mathrm{~cm}$ and a thermal expansion center that coincides with the microscope's optic axis [1]. The detachable sample cartridges have no direct link to the microscope's outside. These two innovations have resulted in drift rates smaller than $1 \mathrm{~nm}$ per hour and sample vibration amplitudes $<0.1 \AA$ r.m.s. [2]. Unfortunately, compared to a side-entry sample rod that supports the sample at one end and exits outside the microscope vacuum at the other end, a detachable cartridge in not as well suited for bringing in "services" to the sample such as cooling, heating, electrical, gases, liquids, straining, tilting, and nano-indenting.

To combine the stability and freedom from drift of our centro-symmetric design with the flexibility of a side-entry sample rod, Nion has developed a new side-entry sample stage. The first version of the stage has been built for Orsay's CHROMATEM project: a high energy resolution monochromated STEM-EELS (HERMES) instrument, whose requirements include cathodo-luminescence (CL) capabilities with both light detection and injection [3], and a liquid nitrogen-cooled sample holder. A CL mirror can be introduced on the entrance side of the sample, in a $6 \mathrm{~mm}$ OL polepiece gap. The mirror is UHV-compatible and precisely moveable, so that the mirror's focal spot can be made to coincide with the "optimum probe formation spot" of the objective lens, about $0.5 \times 0.5 \times 2 \mu \mathrm{m}$ in size (w x d x h). The mirror can couple out CL, or bring in laser illumination, as needed for instance for energy-gain spectroscopy. The cathodo-luminescence capabilities of the system are complemented by Nion's sub-10 meV energy resolution ground-potential monochromator [4], and a new Nion electron energy loss spectrometer optimized for vibrational studies. 
The key features of Nion's side entry stage are:

a) A sample rod with a principal external diameter of $16 \mathrm{~mm}$, small enough so that changes in air pressure outside the column do not result in major sample movement. The rod width is reduced to 8 $\mathrm{mm}$ at the sample, but this is enough to be compatible with a wide variety of sample "services".

b) Nion's ultra-stable sample movement mechanism, which shifts the front of the sample rod via a spherical rolling joint. Motion perpendicular to the rod axis is nearly friction and hysteresis free.

c) Compact airlock and goniometer mechanism that performs just two main functions: vacuum management during sample exchange, and setting the primary (alpha) tilt within a $180^{\circ}$ range with arcminute precision.

d) UHV design principles: all seals are either metal or have a guard vacuum; ion pumps evacuate both the high and the guard vacua; a turbo pump roughs down the airlock; minimal area of the sample rod in high vacuum; and the high-to-guard-vacuum O-rings are never exposed to air (so they don't soak up air moisture and later release it in the high vacuum). The stage can be baked at $140^{\circ} \mathrm{C}$.

Figure 1 shows a schematic cross-section through the inner part of this sample stage. The front of the sample rod is moved by the stage ring in a way that is similar to Nion's cartridge stage design [1]. The motion is coupled into the rod by a spherical rolling joint. The airlock fixes the transverse position of the back of the sample holder, and instabilities affecting the airlock are reduced by $5 \mathrm{x}$ at the sample.

A stable liquid nitrogen holder has been developed by CrIPTA for the stage, and will be described separately. Nion plans to continue working with third parties to develop a variety of in-situ capabilities. The stage's overall performance will be reported at the meeting.

\section{References:}

[1] OL Krivanek et al, Ultramicroscopy 108 (2008) 179.

[2] OL Krivanek et al, Microsc. Microanal. 16 (Suppl 2, 2010) 70.

[3] M Kociak and LF Zagonel, Ultramicroscopy 174 (2017) 50.

[4] OL Krivanek et al., Nature 514 (2014) 209.

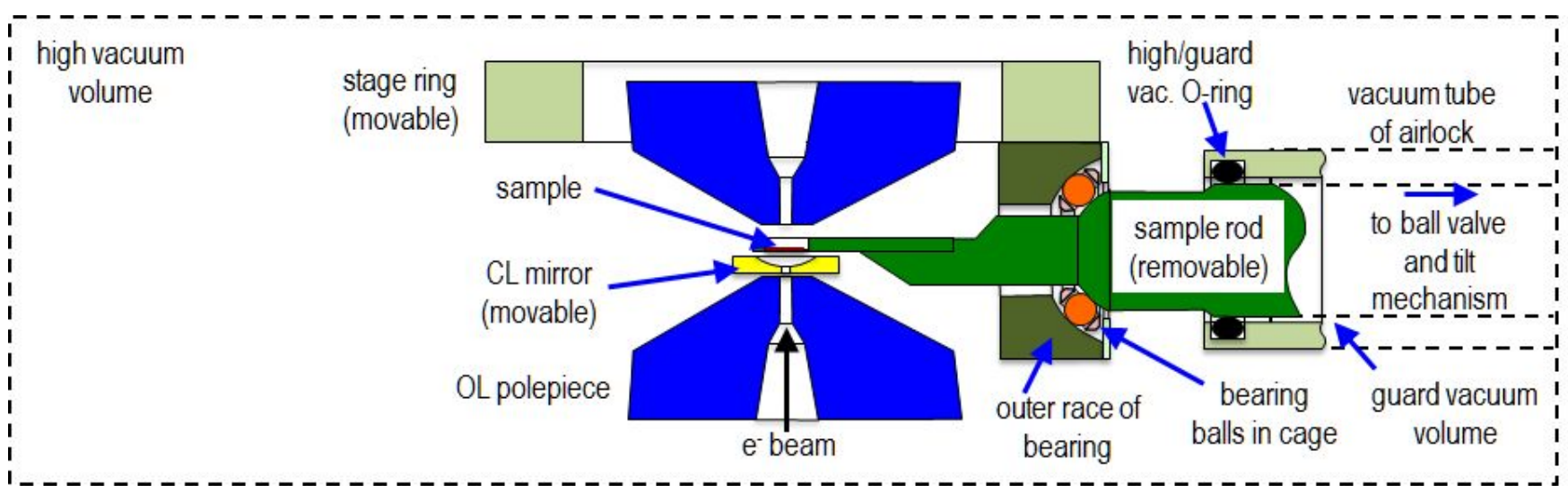

Figure 1. Schematic diagram of the Nion side-entry stage. The front part of the sample rod is moved around by the stage ring. The outer components of the stage are not shown. 